\title{
Experimental Study of the Response of Transonic Diffuser Flow to a Piezoceramic Actuator at Diffuser Throat
}

\author{
Minoru Yaga $^{1}$, Yusuke Uechi ${ }^{2}$, Hiroaki Ozono ${ }^{3}$, Masaaki Ishikawa ${ }^{1}$, Isao Teruya ${ }^{1}$ \\ ${ }^{1}$ Department of Mechanical Systems, Faculty of Engineering, University of the Ryukyus, Okinawa, Japan \\ ${ }^{2}$ Mie Metal Industry Co. Ltd., Mie, Japan \\ ${ }^{3}$ Graduate School of Engineering and Science, University of the Ryukyus, Okinawa, Japan \\ Email: yaga@tec.u-ryukyu.ac.jp
}

Received May 28, 2013; revised June 5, 2013; accepted June 12, 2013

Copyright ( 2013 Minoru Yaga et al. This is an open access article distributed under the Creative Commons Attribution License, which permits unrestricted use, distribution, and reproduction in any medium, provided the original work is properly cited.

\begin{abstract}
An experimental study of the response of a piezoceramic actuator set at the throat to a transonic diffuser is carried out by measuring wall static pressure fluctuations and by visualizing the flow field using schlieren technique. The visualized flow fields are captured with a digital still camera and a digital high speed video camera. The piezo ceramic actuator is attached at the throat of the diffuser and driven by sinusoidal amplified voltage signals. The diffuser used in this experiment is circular arc half nozzle with the height $h^{*}$ and width $w$ of $3 \mathrm{~mm}$ and $25 \mathrm{~mm}$, respectively. The blockage factor of the piezoceramic actuator to the diffuser throat is $9.2 \%$ assuring the effect of change in the throat area rather than the boundary layer disturbances. The piezoceramic actuator is driven at the frequency of $100 \mathrm{~Hz}, 200 \mathrm{~Hz}$, and 300 $\mathrm{Hz}$ and its amplitude is about $1 \mathrm{~mm}$. It is found that the wall static pressure fluctuations and the behavior of the shock wave clearly correspond to the vibration of the piezo ceramic actuator for all the frequency ranges whereas the averaged shock position remains almost unchanged. All the results mentioned above suggest that driving the piezo ceramic actuator at the diffuser throat can be one of the promising techniques to control unsteady transonic diffuser flow.
\end{abstract}

Keywords: Compressible Flow; Shock Wave; Transonic Diffuser; Piezoceramic Actuator; Throat

\section{Introduction}

The unsteady flow field in a transonic diffuser has attracted a great deal of interest not only because of the practical industrial importance but also because of the complexity of the flow itself. It is well known that a shock wave in a transonic flow is basically unsteady due to the interaction between a shock wave and other flow phenomena, i.e., the shock wave oscillation is triggered and maintained by a local interaction between the shock foot and the boundary later developed along the wall surface or upstream and downstream propagating disturbances. Meier [1] reported that shock-induced separation causes the large-amplitude, unstable oscillation of a shock wave. In addition to the shock-induced separation, the effects of propagating disturbances toward the shock waves on the oscillations have been analytically, numerically and experimentally investigated [2,3], including forced oscillations by some functions or rotating rods as a forcing function to drive the shock waves. According to previous studies, several factors affect unsteady shock behavior in transonic diffusers. Therefore, in addi- tion to revealing the causes of the shock oscillations, there have also been attempts to eliminate or reduce these unfavorable unsteady shock oscillations both for internal and external devices. It is quite difficult to deal with these types of oscillations unless the disturbances around the shock wave are attenuated or canceled by some techniques because the longitudinal and boundary layer associated disturbances cannot be separated.

The approach to stabilize the unsteady flow fields is divided into two major controls, which are active control with jets [4,5] and passive control with a porous cavity [6] or vortex generators. These controls are expected to generate the opposite phase of signals or to retard separations of boundary layers. However, the causes of the oscillations include pressure disturbances in a core flow and unsteady phenomena associated with the shockboundary layer interaction, as mentioned above, the future trend for the oscillation controls might be a combination of passive and active controls.

Although the effect of the pressure disturbances in a core flow and disturbances associated with the boundary 
layer should be considered individually in order to clarify the factors affecting the oscillations, both effects cannot be divided because they interact with each other. Thus, in the present paper, we focus primarily on the mechanical disturbances by changing cross sectional area at the throat of a transonic diffuser, which is expected to affect the location of shock waves according to the isentropic flow relations between the throat area and local cross sectional area at a location of the shock wave.

\section{Experimental Apparatus and Procedure}

\subsection{Wind Tunnel Facility}

Figures 1(a)-(c) show the wind tunnel, the detail of the test section, and the driving circuit of the piezoceramic actuator, respectively. The experimental apparatus consists of a $0.7 \mathrm{MPa}$ compressor, a settling chamber, a regulator valve, and a circular arc transonic half diffuser in a blow down wind tunnel discharging to the atmosphere. The throat height of the diffuser and its radius are $\mathrm{h}^{*}=3 \mathrm{~mm}$ and $\mathrm{R}=500 \mathrm{~mm}$, respectively. The span of the diffuser is $33 \mathrm{~mm}$. The flow field in the diffuser is visualized by the schlieren technique and captured with a digital still camera or a digital high-speed video camera, which enables images of the flow field to be captured at a rate of 41,000 frames per second. Unsteady wall static pressure fluctuations are measured using semiconductor pressure sensors at the settling chamber, at the throat, and at positions $x=20,30,40$, and $50 \mathrm{~mm}$ downstream of the throat. The signals from the pressure transducer are digitized at a sampling frequency of $10 \mathrm{kHz}$ with 16-bit accuracy and are analyzed to evaluate the effect of the piezoceramic actuator on the diffuser flow fields. The resonance frequency of the transducers is $50 \mathrm{kHz}$ assuring the accuracy of monitoring the pressure fluctuations. The total pressure at the settling chamber is monitored during the experiments by a computer, which controls the timing of the pressure measurements and flow visualizations.

\subsection{Piezoceramic Actuator}

The piezoceramic actuator is set at the diffuser throat, as shown in Figure 1(b). In order to achieve sufficient displacement by the actuator, a bimorph type piezoceramic

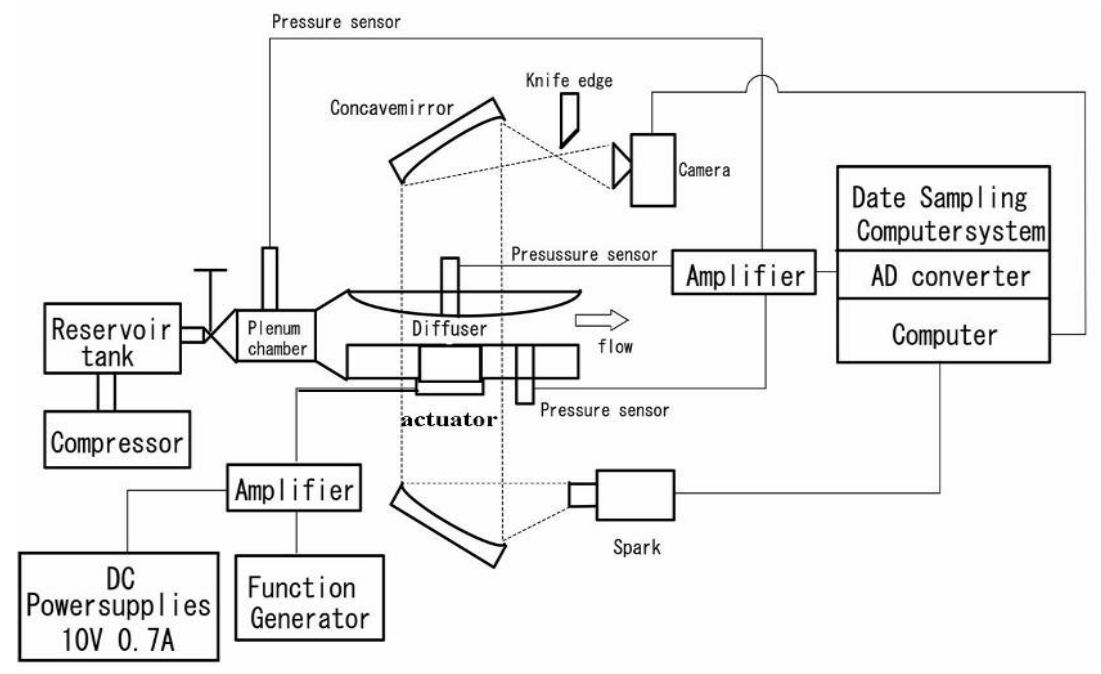

(a)

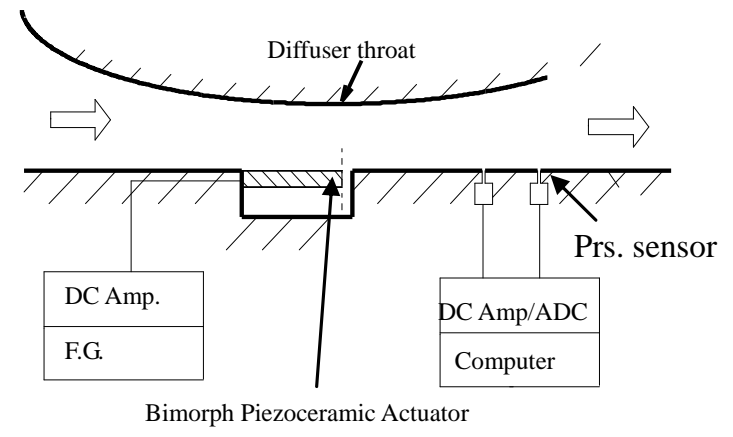

(b)

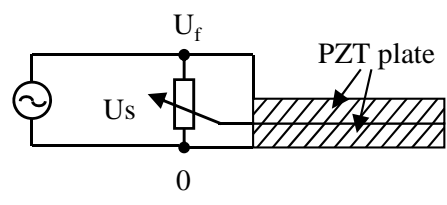

(c)

Figure 1. Experimental apparatus: (a) Wind tunnel and measurement system; (b) Detail of test section; (c) Driving circuit for piezoceramic actuator. 
actuator is adopted and driven by the circuit as shown Figure 1(c). The length, width, and thickness of the piezoceramic actuator are $33 \mathrm{~mm}, 11 \mathrm{~mm}$, and $0.8 \mathrm{~mm}$, respectively. During the experiment, the piezoceramic actuator is connected to a DC type amplified voltage source and then bent in order to change the cross sectional area at the diffuser throat. The maximum input voltage from the signal source, displacement, and natural resonance frequency of the piezoceramic actuator are $60 \mathrm{Vp}, 0.7$ $\mathrm{mm}$, and $400 \mathrm{~Hz}$, respectively. Accordingly, the exciting input frequency to the piezoceramic actuator is limited to $300 \mathrm{~Hz}$. From preliminary experiments, the displacement of the piezoceramic actuator is found to be sufficiently large compared to that with a layer type piezoceramic actuator. In this report, only sinusoidal signals are input into the DC amplifier to achieve simplicity of the frequency analyses.

\section{Experimental Results and Discussion}

\subsection{Results of Flow Visualization}

Figures 2(a)-(d) show typical schlieren images of the flow fields and shock positions for the input frequency of $f_{\mathrm{p}}=200 \mathrm{~Hz}$. It is clear that some quite weak Mach waves are generated near the throat due to the effect of the moving piezoceramic actuator. At the same time, at a pressure ratio of $p_{0} / p_{\mathrm{b}}=1.20$, the starting shock wave appears to be very weak near $x / h^{*}=6.0$, which is followed by some weak shock waves due to the interaction between the shock wave and the boundary layers along the diffuser walls. Although the flow field downstream of the normal shock wave is supposed to be subsonic, Figure 2(a) and subsequent figures illustrate the occurrence of the shock wave downstream of the first normal shock wave.

The second shock wave or multiple shock waves are generated by the acceleration of the main flow due to the change in the effective cross sectional area of the diffuser and the disturbances approaching from downstream of the shock wave. As reported in several previous studies, the shock wave near the throat is unstable, so that the shock wave observed in Figure 2(a) change its position, as will be discussed later herein. Figure 2(b) shows that the slight increase in the pressure ratio causes the downward displacement of the shock wave. Figure 2(c) illustrates the lambda-foot type shock wave on the upper and lower walls due to a typical moderate interaction between the shock wave and the boundary layer. Other shock waves downstream of the first shock position also correspond to the varying throat height. Then, the distance between the throat and the averaged shock positions increases with the pressure ratio. Figures 2(c) and (d) show that the clear shock waves cause boundary layer separation followed by multiple shock waves downstream of the interaction region.

\subsection{Shock Locations for Various Frequencies}

According to Figure 2, the shock waves appear to move gradually in the previous section. Then, it is necessary to check the relation between rough locations and shock waves for various input frequencies of the piezoceramic actuator. Then, their positions are plotted with respect to the pressure ratio and are shown in Figure 3 as a parameter of the input frequencies of $0 \mathrm{~Hz}, 100 \mathrm{~Hz}, 200 \mathrm{~Hz}$, and $300 \mathrm{~Hz}$.

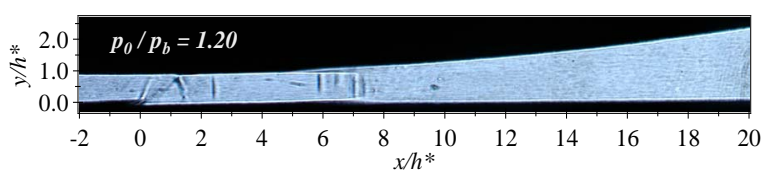

(a)

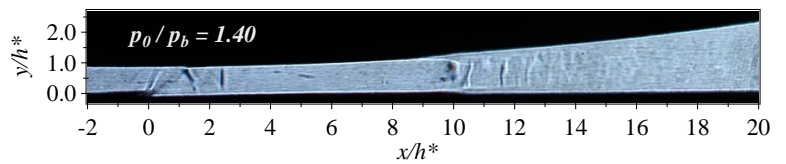

(b)

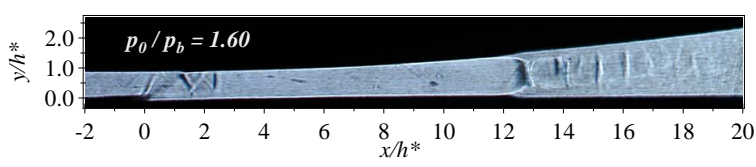

(c)

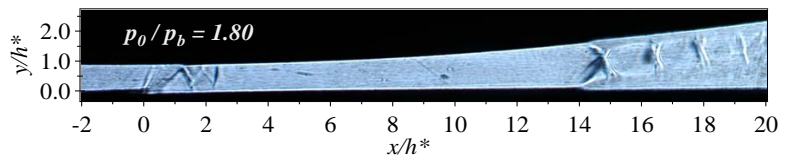

(d)

Figure 2. Schlieren images for frequency $200 \mathrm{~Hz}$ : (a) $p_{0} / p_{\mathrm{b}}=$ 1.2; (b) $p_{0} / p_{\mathrm{b}}=1.4 ;$ (c) $p_{0} / p_{\mathrm{b}}=1.6$; (d) $p_{0} / p_{\mathrm{b}}=1.8$.

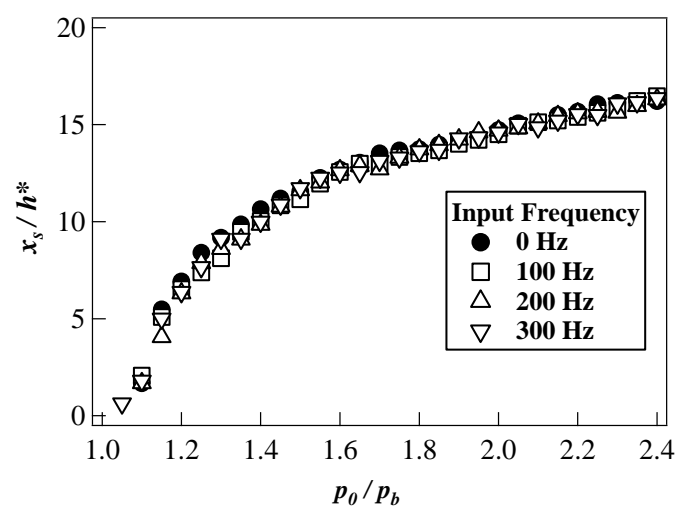

Figure 3. Relation between shock wave position and pressure ratio. 
Figure 3 shows the relation between the positions of shock waves $x_{\mathrm{s}}$ divided by throat height $h^{*}$ and the pressure ratio $p_{0} / p_{\mathrm{b}}$ the shock locations are measured in the schlieren images for various input frequencies of $0 \mathrm{~Hz}$ through $300 \mathrm{~Hz}$. The shock wave is found to move monotonically downstream with the increase in the pressure ratio. However, the gradient of the shock displacement to the pressure ratio decreases with the pressure ratio because the shock-induced separation causes displacement delay. Considering the effect of the piezoceramic actuator, it is easily expected that the location of shock waves corresponds to the throat height because the Mach number just upstream of the shock wave depends on the ratio of the local cross sectional area to the throat area. From the viewpoint of small-scale observation, the shock wave oscillates around its averaged position at the fixed frequency of the piezoceramic actuator, which will be discussed in the following section based on images captured by a high-speed video camera. The behavior of the shock wave is critical to the generation of large pressure fluctuations under the various pressure ratios.

\subsection{Wall Static Pressure Fluctuation}

Figures 4(a)-(d) show the time series pressure fluctuations

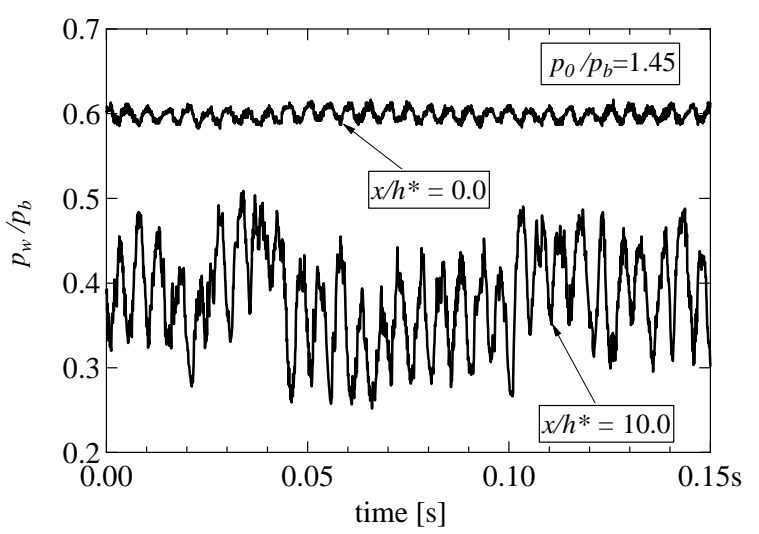

(a)

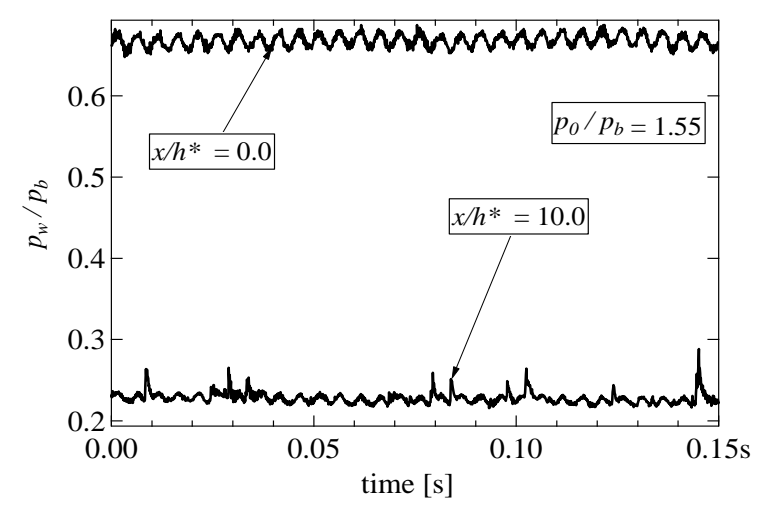

(c) at the throat $x / h^{*}=0.0$ and at $x / h^{*}=10.0$ for a frequency of $200 \mathrm{~Hz}$. The wall static pressure $p_{\mathrm{w}}$ divided by the back pressure $p_{\mathrm{b}}$ at the throat exhibits a clear sinusoidal time history, which is considered to correspond to the behavior of the piezoceramic actuator. On the other hand, the pressure fluctuation at $x / h^{*}=10.0$ indicates a severe large amplitude fluctuation. The large amplitude fluctuation suggests that the shock wave passes back and forth over the measurement position corresponding to the motion of the piezoceramic actuator. The increase in amplitude can be explained by Figure 3, which shows that the shock wave is located around $x / h^{*}=10.0$. Then, in case of a pressure ratio of 1.50 , the shock wave is expected to stand slightly downstream of the pressure measurement position at $x / h^{*}=10.0$.

The influence of the shock wave could reach the pressure measurement position through the subsonic layer in the boundary layer which develops along the diffuser wall. The pressure remains relatively low compared to that shown in Figure 4(a), which indicates that the shock wave stands further downstream than that for $p_{0} / p_{\mathrm{b}}=$ 1.45. However, the effect of the piezoceramic actuator can be still detected. Finally, in the case of $p_{0} / p_{\mathrm{b}}=1.55$, the shock wave moves far downstream of the pressure

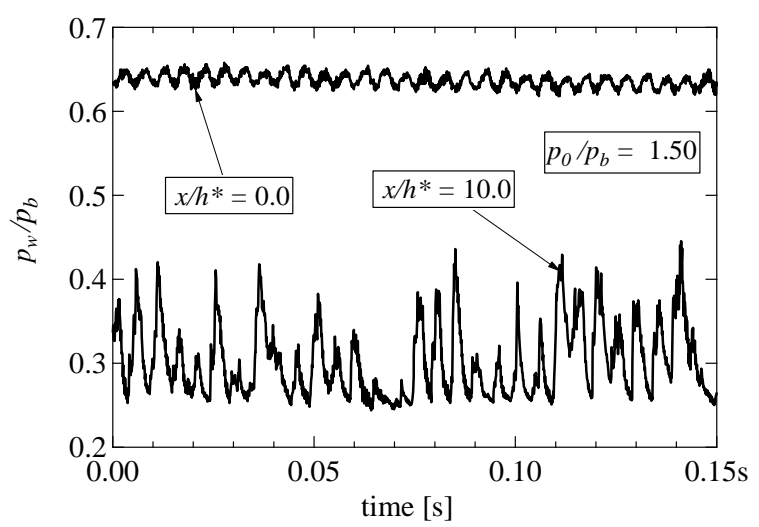

(b)

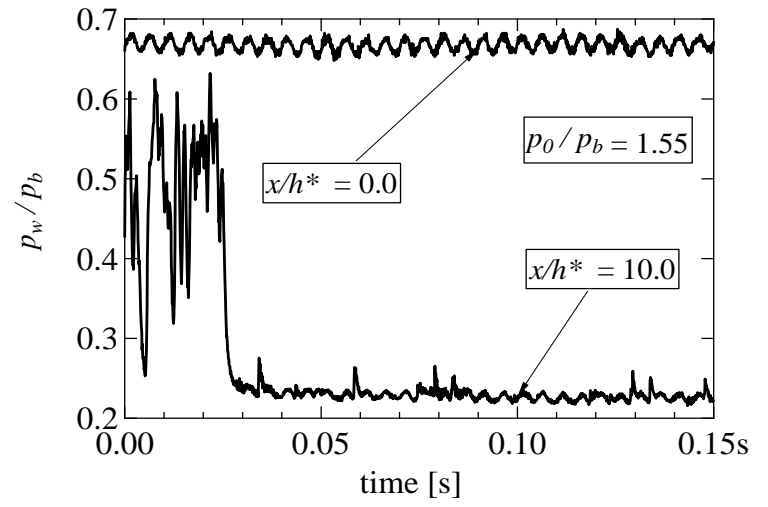

(d)

Figure 4. Wall static pressure fluctuations at throat and $x / h^{*}=10.0$ for input frequency $200 \mathrm{~Hz}:(\mathrm{a}) p_{0} / p_{\mathrm{b}}=1.45 ;(\mathrm{b}) p_{0} / p_{\mathrm{b}}=$ 1.50; (c) $p_{0} / p_{\mathrm{b}}=1.55 ;$ (d) $p_{0} / p_{\mathrm{b}}=1.55$. 
measurement position. This is also confirmed in Figure 3, which showing $x_{s} / h^{*}=10.0$ at a pressure ratio of 1.55 . Both the fluctuating and averaged pressure are much lower due to the downstream displacement of the pressure measurement position. These figures contain information about the shock position and the shock wave behavior. In other words, Figure 4(a) shows the severe pressure fluctuation at $x / h^{*}=10.0$, whereas the fluctuation at the throat does not. This means that the shock wave stands between the throat and the measurement position at $x / h^{*}=10.0$. As the pressure ratio increases, the fluctuation at $x / h^{*}=10.0$ gradually decreases the pressure fluctuation, achieving the smallest pressure fluctuation, as shown in Figure 4(c). The pressure fluctuation as shown Figure 4(c) denotes the supersonic state at $x / h^{*}=10.0$.

However, the sudden change in the average pressure was observed as shown in Figure 4(d) under the same condition, which is caused by the flow separation downstream of the shock wave. The increase in the pressure fluctuation indicates the shock wave displacement upstream due to the flow separation. However, some time after the increase in pressure, the average value and fluctuation decrease, denoting the shock wave located downstream of the position at $x / h^{*}=10.0$. The pressure fluctuations at the throat are found to oscillate sinusoidally for all pressure ratios. Figure 4(d) also reveals that the wall pressure fluctuation at a pressure ratio of 1.55 suddenly changes its level at time $t=0.03 \mathrm{~s}$, which suggests that the flow has just recovered from an unexpected separation downstream of the shock wave.

\subsection{Root Mean Square of the Pressure Fluctuation}

It is well known that wall static pressure fluctuations greatly decrease when a flow becomes supersonic. In other words, monitoring the wall static pressure fluctuations clarifies whether the flow is supersonic or subsonic. In the previous section, the pressure fluctuations also indicate the rough shock wave positions. Then, for a more quantitative evaluation of the pressure fluctuations, the root mean square of pressure fluctuations is one of the indices of the shock wave position and its behavior.

Figures 5(a)-(d) show the root mean squares $p_{\text {rms }}$ divided by the atmospheric pressure $p_{\mathrm{b}}$ for three different frequencies $100 \mathrm{~Hz}, 200 \mathrm{~Hz}$, and $300 \mathrm{~Hz}$ and compared to the case for $0 \mathrm{~Hz}$ as a reference. Figure 5(a) shows that the root mean square $p_{\text {rms }} / p_{\mathrm{b}}$ for the input frequency of $f_{\mathrm{p}}=0 \mathrm{~Hz}$ at the throat shows almost no increase in $p_{\text {rms }} / p_{\mathrm{b}}$ with the increase in the pressure ratio $p_{0} / p_{\mathrm{b}}$. On the other hand, activation occurs at the throat, the rms increases with the pressure ratio. This indicates that the piezoceramic actuator always affects the pressure fluc-

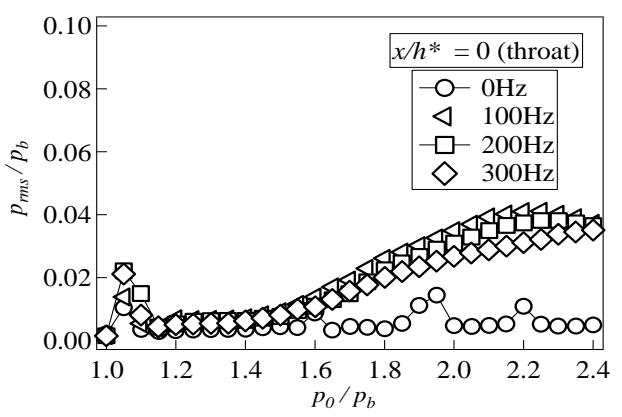

(a)

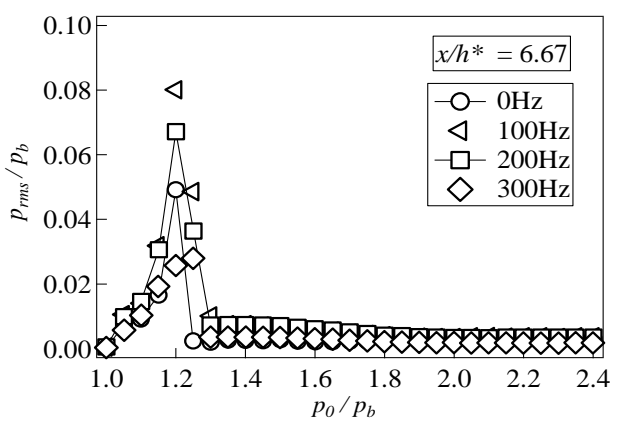

(b)

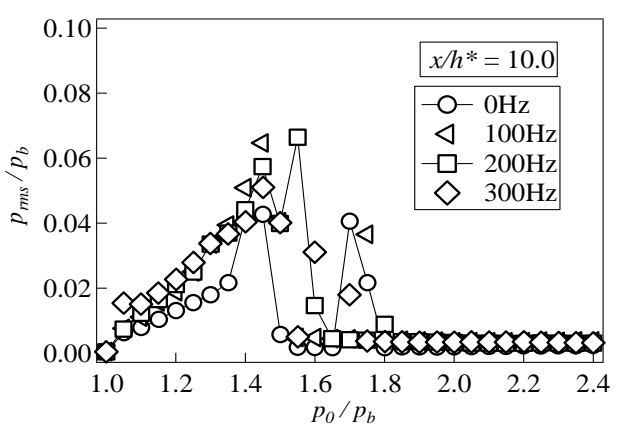

(c)

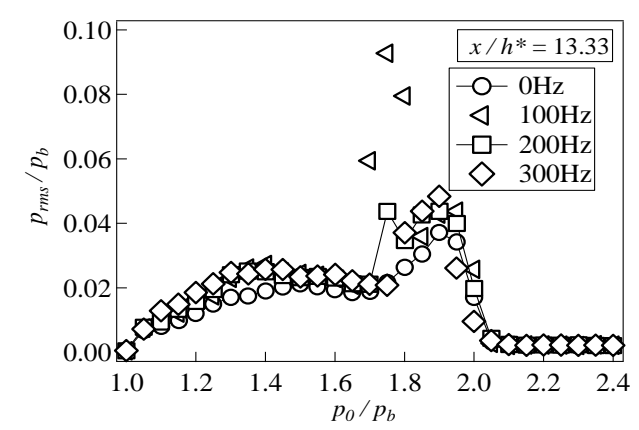

(d)

Figure 5. RMS of wall static pressure fluctuations: (a) $x / h$ * $=0.0$; (b) $x / h^{*}=6.67$; (c) $x / h^{*}=10.0$; (d) $x / h^{*}=13.33$.

tuation at the throat. When the rms reaches approximately zero, the state of the measurement position is considered to be supersonic. This indicates that the shock wave is located downstream of the measurement position. 
In Figure 5(b), the clear peaks at a pressure ratio $p_{0} / p_{\mathrm{b}}$ of approximately 1.2 show that a single and relatively weak shock wave appears and moves downstream with the increase in the pressure ratio. The sudden drop in the rms indicates the completion of the process from the subsonic-to-supersonic transition due to the downstream displacement of the shock wave. This process is independent of the input frequencies of the piezoceramic actuator.

Figure 5(c) shows that the rms at a pressure ratio of approximately 1.7 suddenly increases, which is caused by the unexpected separation of a boundary layer, as mentioned in the previous section. This also indicate the shock wave existence around the position of $x / h^{*}=10.0$. However, for a pressure ratio greater than 1.8, the rms for all input frequencies becomes approximately zero, which suggests that the flow is completely supersonic. Note that the source of the signal originates at the throat, and then even if the flow measurement position is supersonic, the signal from the throat can be detected. Figure 5(d) indicates the same variations that is all the rms for each input frequency suddenly decrease at the pressure ratio at 2.0 denoting the supersonic state at this postion.

\subsection{FFT Analyses of Wall Pressure Fluctuation}

The rms of the pressure fluctuation usually becomes large when the oscillating shock wave approaches the monitoring position. It is also important to examine the unsteady behavior of the shock wave in detail, especially in this case, in order to confirm the response of the flow field to the piezoceramic actuator.

One of the best ways to evaluate the effect of the actuation is to check the contributions of every frequency by means of FFT analysis of the pressure fluctuations at $x / h^{*}=6.67$. Figures 6(a)-(d) show the results of the FFT analysis of the pressure fluctuations for each pressure ratio. Figure 6(a) shows no dominant frequency and almost no frequency output for pressure ratios greater than $p_{0} / p_{\mathrm{b}}=1.25$, as deduced from the results for the rms shown in Figure 5(b). This indicates that the shock wave oscillates with no dominant frequency for all of the pressure ratios and that the state of the flow at $x / h^{*}=$ 6.67 becomes supersonic at pressure ratios greater than 1.25. Moreover, the levels of each spectrum are not so large because the shock wave at this position is not so strong.

On the other hand, Figures 6(b)-(d) clearly show the dominant frequencies for each pressure ratio, which corresponds to the input frequency to the piezoceramic actuator. Note that even in the supersonic state, the frequency of $200 \mathrm{~Hz}$ can be observed. These figures indicate the effect of the piezoceramic actuator on the flow fields. In addition to the results of the visualization,

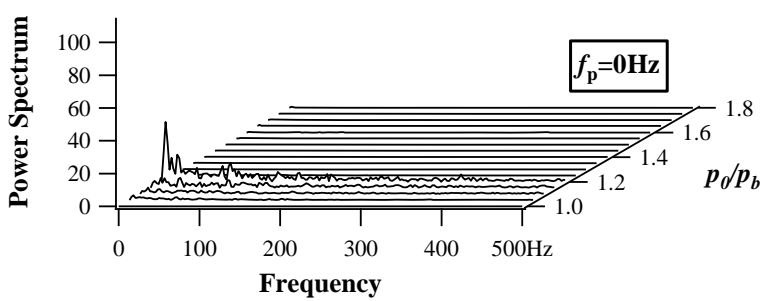

(a)

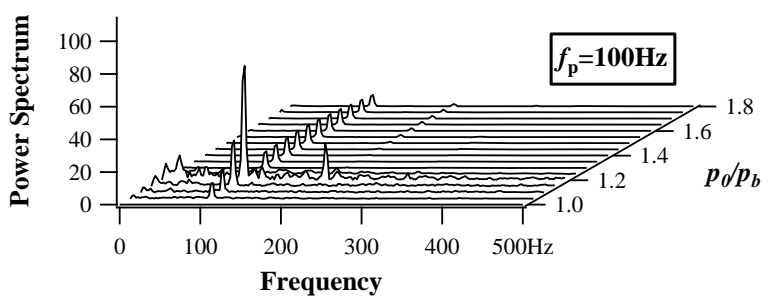

(b)

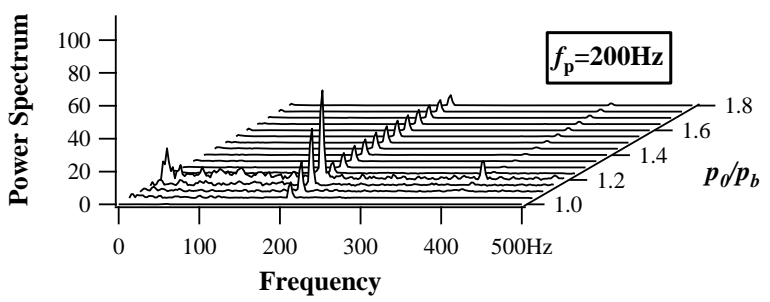

(c)

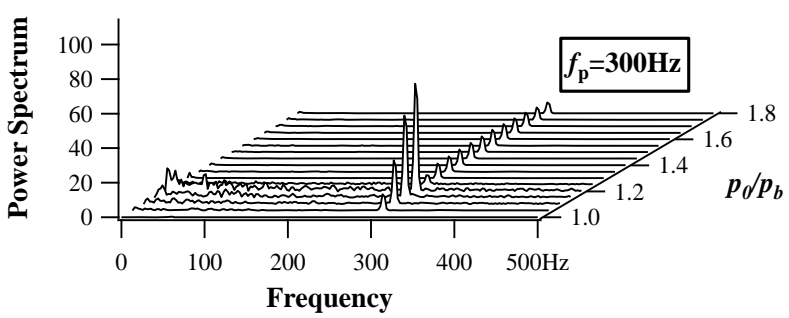

(d)

Figure 6. FFT analysis of wall static pressure fluctuations at $x / h *=6.67$ : (a) $f_{\mathrm{p}}=0 \mathrm{~Hz}$; (b) $f_{\mathrm{p}}=100 \mathrm{~Hz}$; (c) $f_{\mathrm{p}}=200 \mathrm{~Hz}$; (d) $f_{\mathrm{p}}=300 \mathrm{~Hz}$.

monitoring the wall static pressure fluctuation is a quantitative way to confirm the effect of the actuator.

Figures 7(a)-(d) show the FFT analysis of the pressure fluctuation at $x / h^{*}=10.0$ for the same input frequency as that in Figure 6. Figure 7(a) shows no dominant frequency but only a relatively low frequency, which has the same tendency as that in Figure 6(a). Figures 7(b)-(d) show clear dominant frequencies. This indicates the possibility of reducing the pressure fluctuation by controlling the shock position, which is expected to cancels the pressure fluctuation. In the figures, a wide range of frequencies are observed at a pressure ratio of approximately 1.7 due to the unexpected shock-induced separation of the boundary layer. All of the figures show a sudden decrease in the power spectrum at a pressure ratio of approximately 1.4. This implies that the shock 


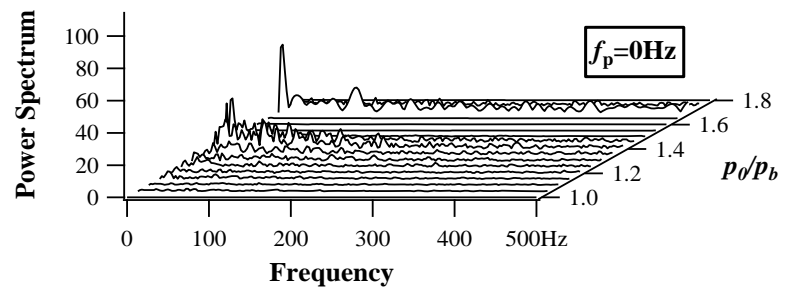

(a)

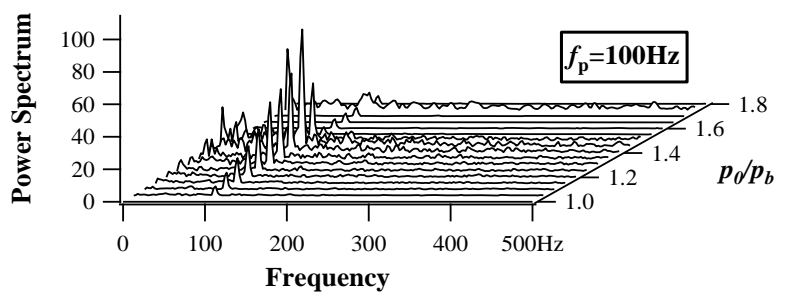

(b)

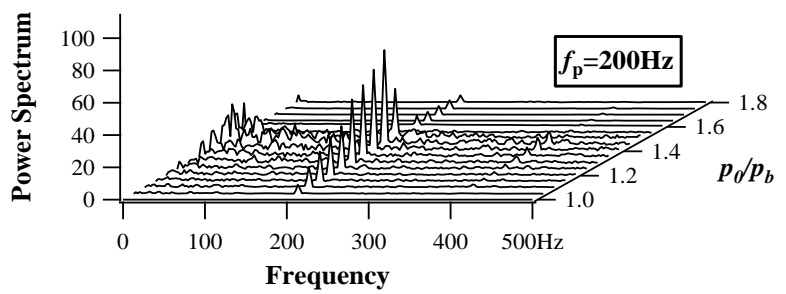

(c)

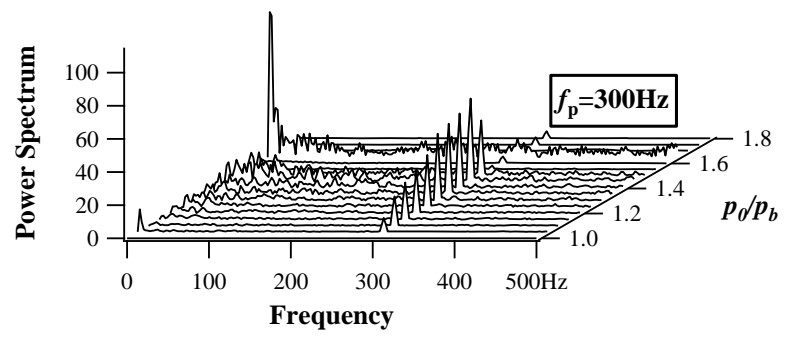

(d)

Figure 7. FFT analysis of wall static pressure fluctuations at $x / h^{*}=10.0$ : (a) $f_{\mathrm{p}}=0 \mathrm{~Hz}$; (b) $f_{\mathrm{p}}=100 \mathrm{~Hz}$; (c) $f_{\mathrm{p}}=200 \mathrm{~Hz}$; (d) $f_{\mathrm{p}}=300 \mathrm{~Hz}$.

wave completely passes the measurement position, resulting in a change in state from subsonic to supersonic.

\subsection{FFT Analysis of Shock Positions}

The wall static pressure fluctuations provide us with only local information on the shock wave behavior around the measurements positions. Accordingly, in order to clarify the entire flow field, the variations in unsteady shock positions are also important because the shock wave always contains all of the information on its upstream and downstream conditions regardless of its position.

Figures 8(a)-(d) show the results of the FFT analyses of the shock positions under the influence of the piezoceramic actuator as well as with no control. In case of no control, there is no dominant frequency, as shown in

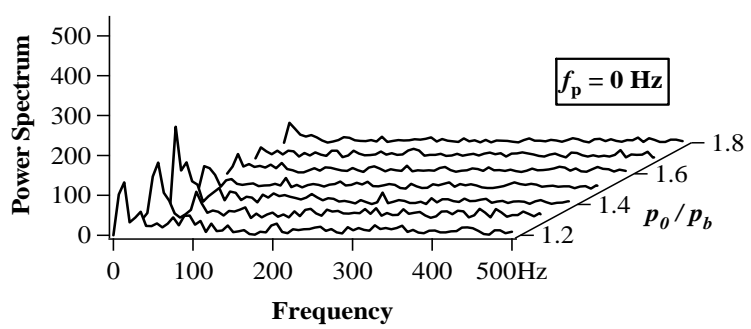

(a)

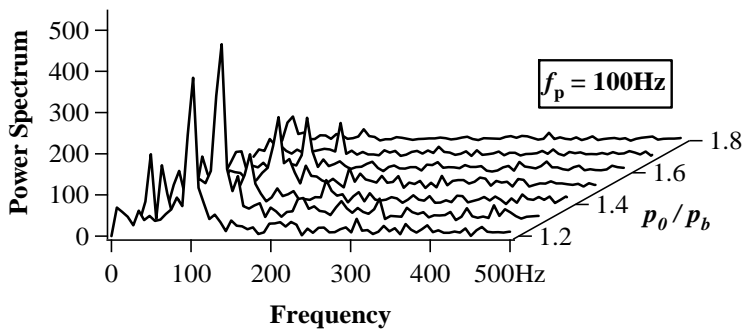

(b)

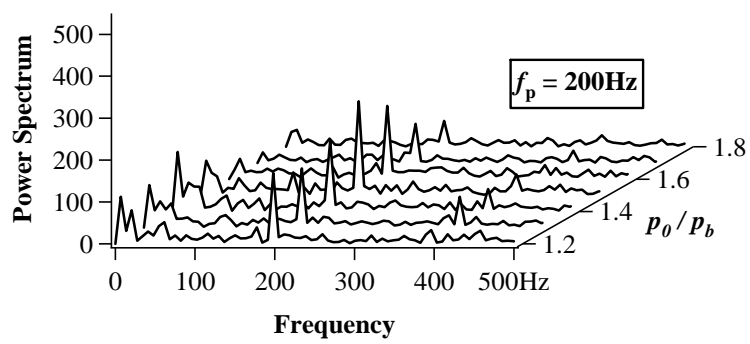

(c)

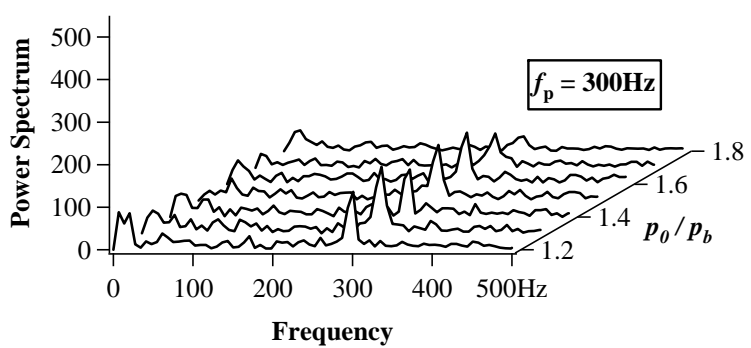

(d)

Figure 8. FFT analysis of Shock positions: (a) $f_{\mathrm{p}}=0 \mathrm{~Hz}$; (b) $f_{\mathrm{p}}=100 \mathrm{~Hz}$; (c) $f_{\mathrm{p}}=200 \mathrm{~Hz}$; (d) $f_{\mathrm{p}}=300 \mathrm{~Hz}$.

Figure 8(a). The relatively low-frequency spectrum is observed as long as the pressure ratio is smaller than 1.4. This shows that the shock wave passes the measurement position monotonically as the pressure ratio increases. In contrast, for a shock wave frequency of $100 \mathrm{~Hz}$, a dominant frequency is observed until a pressure ratio of 1.5. The peak value of the dominant frequency decreases as the pressure ratio increases. This decrease in the peak value of the dominant frequency is explained by the behavior of the shock wave, as shown in Figure 3. That is, the gradient of the shock wave displacement decreases as the pressure ratio increase as shown in Figure $\mathbf{3}$ due to the expansion rate of the cross sectional area of the diffuser. 
This implies that shock wave located downstream is insensitive to the pressure fluctuation compared with that located upstream position. Then, the oscillation of the shock wave gradually decreases as it moves downstream. The diminishing of the dominant frequency for higher pressure ratios might be due to the relative increase in other frequencies.

On the other hand, when the higher frequency is applied to the throat, the clearly peaky dominant frequency, which is the exact same frequency as that input to the piezoceramic actuator, can be observed. Note also that the applied frequency remains, even for the higher pressure ratio, which suggests that the shock wave is always under the influence of the piezoceramic actuator. The tendencies observed in Figure 8(c) also appear in Figure 8(d). In other words, the input frequency of $300 \mathrm{~Hz}$ is the dominant frequency for all pressure ratios. Then, actuation at the throat is a promising method for controlling shock wave behavior.

\section{Conclusions}

A piezoceramic actuator is applied to the throat of a circular arc diffuser with various driving frequencies in order to clarify the response of the flow field and shock wave behaviors to the piezoceramic actuator. The piezoceramic actuator, which moves periodically as a reference signal, is considered to be a driving force for oscillation phenomena. The conclusions are summarized as follows:

1) The starting shock wave moves downstream monotonically with the increase in the wind tunnel pressure ratio, regardless of the input frequencies to the piezoceramic actuator.

2) The rms values of the wall pressure fluctuations decrease suddenly just after the shock wave completely passes over the measurement position for all driving fre- quencies.

3) The pressure fluctuations at the throat and downstream of the throat correspond to the exact same frequencies of the input frequencies to the piezoceramic actuator in both cases that the position is supersonic and subsonic state.

4) Shock wave behaviors are also confirmed to correspond to the piezoceramic actuator behaviors.

\section{REFERENCES}

[1] G. E. A. Meier, "Shock Induced Flow oscillations in a Laval Nozzle,” In: K. Oswatitsch and D. Rues, Eds., Symposium Transonicum II, Springer, Berlin, 1976, pp. 252-261.

[2] T. Hsieh, T. J. Bogar and T. J. Coakley, "Numerical Simulation and Comparison with Experiment for SelfExcited Oscillations in a Diffuser Flow,” AIAA Journal, Vol. 25, No. 7, 1987, pp. 936-943. doi:10.2514/3.9725

[3] R. Bur, R. Benay, A. Galli and P. Berthouze, "Experimental and Numerical Study of Forced Shock-Wave Oscillations," Aerospace Science and Technology, Vol. 10, No. 4, 2006, pp. 265-278. doi:10.1016/j.ast.2005.12.002

[4] E. Benini, R. Biollo and R. Ponza, "Efficiency Enhancement in Transonic Compressor Rotor Blades Using Synthetic Jets: A numerical Investigation,” Applied Energy Vol. 88, No. 3, 2011, pp. 953-962, doi:10.1016/j.apenergy.2010.08.006

[5] P. B. Salunkhe, J. Joseph and A. M. Pradeep, “Active Feed-Back Control of Stall in a Axial Flow Fan under Dynamic Inflow Distortion,” Experimental Thermal and Fluid Science, Vol. 35, No. 6, 2011, pp. 1135-1142. doi:10.1016/j.expthermflusci.2011.03.008

[6] M. Yaga, T. Haga and K. Oyakawa, "Study on Passive Control in a Transonic Diffuser,” AIAA Paper 2000-0902, 38th Aerospace Sciences Meetings \& Exhibit, Reno, January 2000, pp. 1-11. 